\title{
Option Strangles: An Analysis of Selling Equity Insurance
}

\author{
Clemens Kownatzki \\ Pepperdine University \\ Hisam Sabouni \\ Claremont Graduate University
}

We analyze the full return characteristics of option strangles and develop a set of models to help investors avoid getting steam-rolled. Our results show that selling SPY strangles are generally profitable across all time frames and 'widths.' Our model posted the largest average returns of $18.28 \%$ in 2009 followed by $16.85 \%$ in 2011. We find new evidence on maximum drawdowns which indicates that losses on some positions can be the equivalent of the profits gained on approximately forty prior positions. This payoff profile has given rise to the metaphor of selling option contracts as the equivalent of "picking up nickels in front of a steam roller."

Keywords: Derivatives, Volatility, Options, Option Pricing, Black-Scholes-Merton

\section{INTRODUCTION}

The finance literature on derivatives is full of studies on the pricing of options, estimating volatility and developing models to forecast crises and market crashes. However, to our knowledge, there has been much less attention on the merits of specific option strategies, in particular those of shorting option straddles and strangles. We aim to provide some new insights by examining the merits of shorting option strangles on the popular SPDR S\&P 500 Exchange Traded Fund (SPY) over the period of 2005 to 2018. SPY is the most liquid exchange traded fund in the United States with an average daily volume of about 500,000 contracts (“"CBOE: Individual Index and ETF Volume Reports"” 2017).

The price of an option is dependent on the breadth of possible scenarios that may occur over the life of an option. Canonical models, such as that of Merton (1973) highlight the relative importance of volatility in determining the price of an option. As individuals' perceptions of volatility change over time, the price of options can vary dramatically. In a market of risk-averse investors, participants may be willing to overpay for insurance (Ederington and Guan (2002), Dichtl and Drobetz (2011)). Therefore, one could argue that options, which act as insurance, may be consistently overpriced and if they are overpriced, strategies of shorting options irrespective of market trend may be profitable. Furthermore, options have expirations and become worthless if they expire out-of-the-money. As a result, their time values decline exponentially as they approach expirations (theta decay). The combination of these factors motivates us to analyze the non-directional approach of shorting options at various strike prices and expirations. More precisely, we analyze the strategy of shorting strangles, that is the simultaneous sale of out-of-the-money calls and out-of-the-money put options on the same underlying asset and the same 
expiration date. In our studies, we aim to provide empirical evidence of the profitability of these options strategies and to establish a classification of optimal trades from a risk-return perspective.

Although there is extensive literature on derivatives, pricing of options, estimating volatility and related phenomena, to our knowledge, only few researchers focused on examining the rationale as well as the benefits and nature of returns on specific option strategies, in particular those of shorting option strangles. Similar statements have been expressed by Coval and Shumway (2001), Bollen and Whaley (2004), and Chaput and Ederington (2005).

Professional option traders tend to be net-sellers of options by taking advantage of these expensive option premiums. The well-documented history of Long-Term Capital Management (LTCM) is case in point. Lowenstein (2000) notes that LTCM usually took the other side of the trade of option buyers who wanted insurance against a market decline.

We are motivated to examine the benefits of shorting strangles and hypothesize that options are richly priced in normal market conditions. Coval and Shumway (2001) examine zero-beta, i.e. delta-neutral straddle positions and find that at-the-money long straddle positions produce average losses of approximately three percent per week. By implication, they suggest that shorting delta-neutral straddle positions have positive weekly returns. Bondarenko (2014) argues that specifically one-month S\&P 500 put options were "grossly overpriced." Bollen and Whaley (2004) concur that abnormal simulated trading profits arise from shorting options, however, they propose that the implied volatility from traded option prices is greater than realized volatility because of a "volatility markup" that compensates market makers in options for the cost of operations. Although they concede that option premiums are "considerably higher" than prices derived from the Black-Scholes formula and realized market volatility, they argue that this overpricing does not present profitable arbitrage opportunities net of cost and associated risk.

While we do not consider arbitrage opportunities for a market maker, we would argue that the considerable over-pricing of options constitutes an opportunity to derive returns over and above the riskfree rate. Simon (2007) reports that shorting QQQ straddles \& strangles without additional conditions is significantly profitable when time horizons are 10 business days even after factoring in transactions costs. Kownatzki (2016) shows that the VIX, as a proxy for implied volatility, systematically overestimates actual volatility in non-crisis periods and that option traders tend to be net-sellers of these overpriced options for the same reasons stated above.

Our results suggest, selling SPY strangles are generally profitable across a variety of widths. However, the payoff profile of a short option strangle exposes the contract seller to a potential for unlimited losses. Our evidence on maximum drawdowns indicates that losses on some positions can be the equivalent of the profits gained on approximately forty prior positions. This payoff profile has given rise to the metaphor of selling option contracts as the equivalent of "picking up nickels in front of a steam roller." The goal of our paper is to analyze the full return characteristics of option strangles and to develop a set of models to help investors avoid getting steam-rolled. We use information that is available at the time a position is under consideration to predict the profitability of strangles and to avoid strangle positions with potentially large losses.

\section{METHODOLOGY}

Few papers had a greater impact on finance theory than the seminal papers by Black and Scholes (1972) and Merton (1973). Their ground-breaking work on the pricing of European-style options is commonly referred to as the Black-Scholes-Merton (BSM) model. Although the model is relying on perhaps some unrealistic assumptions, the intuition and mathematical beauty of their closed-form solution may be a major reason for its widespread use until today. The model prices a European call and put as follows:

Call $=S_{0} e^{-q T} N\left(d_{1}\right)-K e^{-r T} N\left(d_{2}\right)$ 


$$
\begin{gathered}
\text { Put }=K e^{-r T} N\left(-d_{2}\right)-S_{0} N\left(-d_{1}\right) e^{-q T} \\
\qquad d_{1}=\frac{\ln \left(\frac{S_{0}}{K}\right)+\left(r f-q+\frac{\sigma^{2}}{2}\right) T}{\sigma \sqrt{T}} \\
d_{2}=d_{1}-\sigma \sqrt{T}
\end{gathered}
$$

where, $S_{0}$ is the spot price of the underlying security, $q$ is the continuously compounded dividend yield, $N(\cdot)$ represents the cumulative normal distribution, $K$ is the strike price of the option, $r$ is the risk-free rate, $\sigma$ is the volatility of the underlying security, and $T$ is the time to expiration expressed in years.

In the Black-Scholes-Merton framework, $N\left(d_{2}\right)$ represents the risk-neutral probability of the underlying securities price at expiration $\left(S_{T}\right)$ exceeding the strike price $\left(K_{c}\right)$ (i.e. $\operatorname{Prob}\left(S_{T}>K_{c}\right) \approx N\left(d_{2}\right)$ ).

In the case of a put option, $N\left(-d_{2}\right)$ represents the risk-neutral probability of the underlying securities price at expiration $\left(S_{T}\right)$ falling below the strike price $\left(K_{p}\right)$ (i.e. $\operatorname{Prob}\left(S_{T}<K_{p}\right) \approx N\left(-d_{2}\right)$ ).

A derivatives trader is more interested in the likelihood of profitability when taking on a position than the likelihood of the underlying security's price exceeding or falling below the strike price(s), because the likelihood of profitability represents the true break-even odds on the position. A rational individual will be indifferent between the two measures, whereas a behavioral agent that may distort probabilities, will prefer the measure of the likelihood of profitability (Tversky and Kahneman, 1992). We extend the Black-Scholes-Merton framework to estimate the likelihood of profitability for a call option by including the price of the option collected, or, paid $P_{c}$ :

$d_{1}^{\prime}=\frac{\ln \left(\frac{S_{0}}{\left(K+P_{C}\right)}+\left(r f-q+\frac{\sigma^{2}}{2}\right) T\right.}{\sigma \sqrt{T}}$

$d_{2}{ }^{\prime}=d_{1}{ }^{\prime}-\sigma \sqrt{T}$

With this slight modification, $N\left(d_{2}{ }^{\prime}\right)$ represents the risk-neutral probability of the underlying securities price at expiration exceeding the strike price plus the price of the option (i.e. $\operatorname{Prob}\left(S_{T}>(K+\right.$ $\left.\left.P_{c}\right)\right) \approx N\left(d_{2}{ }^{\prime}\right)$ ), which is a measure of the likelihood of profitability for a call option purchaser and the likelihood of loss for a call option seller. For put options, we can estimate the likelihood of profits in a similar manner by including the price of the put option $P_{p}$ :

$d_{1}^{\prime \prime}=\frac{\ln \left(\frac{s_{0}}{(K-P p)}+\left(r f-q+\frac{\sigma^{2}}{2}\right) T\right.}{\sigma \sqrt{T}}$

Here $N\left(-d_{2}{ }^{\prime \prime}\right)$ measures of the likelihood of profitability for a put option purchaser and the likelihood of loss for a put option seller. Using this methodology, the likelihood of profitability of more complex strategies that combine call and put options, such as ashort (long) strangle position where one call is sold (purchased) and one put is sold (purchased) of equal distance from the prevailing price at time 0 , can also be estimated. Using the strangle example:

$d_{1}^{\prime \prime \prime}=\frac{\ln \left(\frac{s_{0}}{\left(K+P_{c}+P p\right)}+\left(r f-q+\frac{\sigma^{2}}{2}\right) T\right.}{\sigma \sqrt{T}}$

$$
d_{2}{ }^{\prime \prime \prime}=d_{1}{ }^{\prime \prime \prime}-\sigma \sqrt{T}
$$


$d_{1}^{\prime \prime \prime \prime}=\frac{\ln \left(\frac{s_{0}}{\left(K-P_{c}-P p\right)}+\left(r f-q+\frac{\sigma^{2}}{2}\right) T\right.}{\sigma \sqrt{T}}$

$$
d_{2}{ }^{\prime \prime \prime \prime}=d_{1}{ }^{\prime \prime \prime \prime}-\sigma \sqrt{T}
$$

The likelihood of profitability for a short-strangle is therefore $2-N\left(d_{2}{ }^{\prime \prime \prime}\right)-N\left(-d_{2}{ }^{\prime \prime \prime \prime}\right)$ and the likelihood of profitability for a long-strangle is $N\left(d_{2}{ }^{\prime \prime \prime}\right)+N\left(-d_{2}{ }^{\prime \prime \prime \prime}\right)$.

In our data, we define a profitable strangle as one that has a profit greater than zero. Our baseline specification is our simple extension to the BSM framework to determine the likelihood of strangle profitability. We compare the set of profitable strangles in our test set against our extensions of the BSM framework. A strangle is predicted as profitable if it has a likelihood of profitability that is greater than the average likelihood of profitability in our training sample ${ }^{1}$.

Our regression based models take in all of the inputs of the BSM framework and seek to predict the likelihood of profitability. The estimated models are fitted using the training data and compared to the BSM model in our test set. The aim of our model estimation is two-fold: (1) capture well documented option market anomalies such as the volatility smile and (2) capture the features of options contracts that are ideal for shorting.

\section{Motivation}

Professional option traders are on balance net-sellers of option contracts, often intuitively taking advantage of theta decay of option premiums. As time to expiration decreases, the price of a call (put) option decreases exponentially. This exponential decay is declared through the Greek letter. In the BSM framework, it is the partial derivative of the call (put) option with respect to time to expiration:

Call Theta: $\frac{\partial C}{\partial T}=-S_{0} N^{\prime}\left(d_{1}\right) \frac{\sigma e^{-q t}}{2 \sqrt{T}}+q S_{0} N\left(d_{1}\right) e^{-q T}-r K e^{r T} N\left(d_{2}\right)$

Call Theta: $\frac{\partial C}{\partial T}=-S_{0} N^{\prime}\left(d_{1}\right) \frac{\sigma e^{-q t}}{2 \sqrt{T}}+q S_{0} N\left(d_{1}\right) e^{-q T}-r K e^{r T} N\left(d_{2}\right)$

We are motivated to explore the profitability of option trading from a similar perspective. In an efficient market, where financial instruments are priced under no-arbitrage conditions, a non-directional option strategy such as a short-strangle should be able to take advantage of the theta decay in option premiums. Hence the colloquialism often heard on trading floors: "When you are shorting an option, time is your friend but when you're buying an option, time is your enemy."

The exponential theta decay is easy to spot with a graph that shows theta values on the $y$-axis and time to expiration on the $\mathrm{x}$-axis. 


\section{FIGURE 1}

\section{EVOLUTION OF THETA DECAY AS IMPLIED BY THE BLACK-SCHOLES MODEL}

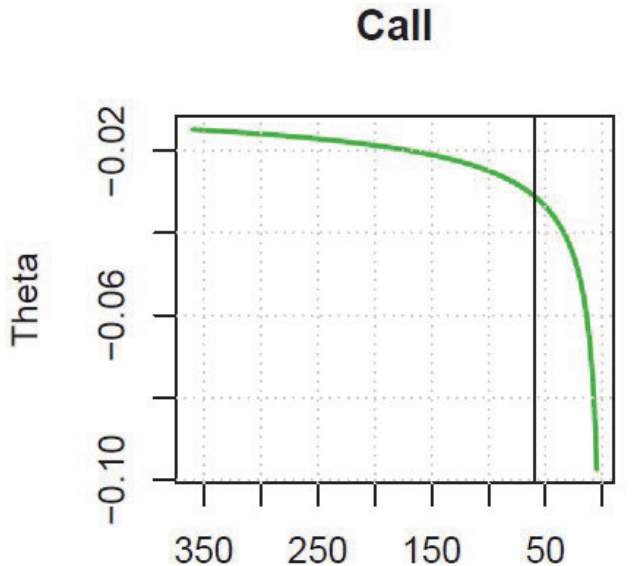

Days to Expiration

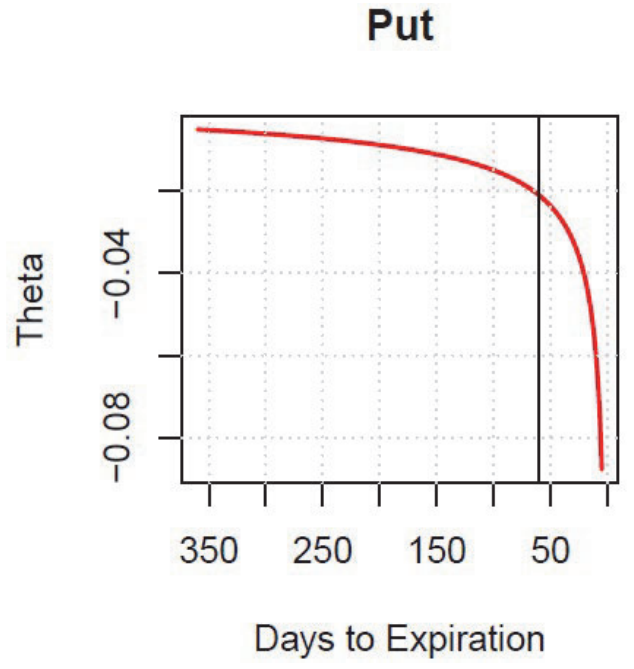

Put

Note: Evolution of theta decay as implied by the Black-Scholes model. Theta begins to exponentially decay around 60 days to expiration, which is depicted by a vertical line in each chart.

Is there an optimal time frame to take advantage of theta decay? Figure 1 depicts how theta values drop off as time to expiration decreases. Theta values are calculated based on a set of generic Call and Put equity options using the BSM model with the following input parameters: $S_{0}=100, K=100, \sigma=$ $20 \%$, and $r f=3 \%$.

A first visual examination of the theta behavior suggests that theta values begin a steeper descent around 50 days to expiration and the values continue to decline exponentially thereafter. To take advantage of the steeper slopes, we examine short strangles with 40-60 days to expiration. Li, French and Chen (2017) propose a similar time frame and suggest that informed option traders prefer medium maturity of 30-90 days. In practical terms, we need to balance the amount of option premium with the theta decay. Although an option with say 5 days to expiration has a much lower theta value, the amount of premium collected is also much smaller. Furthermore, as option sellers we would be exposed to much greater gamma risk, since gamma increases as we get closer to expiration.

\section{Data}

Our data consists of daily options prices from iVolatility.com, a leading provider of historical options data. Our sample period covers the period of January 2005 to November $2018^{2}$. Throughout our analysis, we use the prevailing options bid and ask prices as of 3:45 PM EST to assure market accessibility. The data from iVolatility include estimates of all greeks over time (delta, gamma, vega, rho, theta, and implied volatility), which we rely upon across all of our specifications. The data were filtered by maintaining contracts that have a positive open interest, a mid-point price greater than zero, and an expiration date within 40 to 60 days. The filtered data are used to generate option strangle positions of widths ranging from $1 \%$ to $15 \%{ }^{3}$. That is, for each day in our sample we find all options that were available for trading after our first set of filters that have a strike price within a given width (e.g. 1\% above or below the price of the underlying as of 3:45 pm EST). Of the available contracts on each day, we select one call and one put option that have the longest amount of time to expiration within a given width. All characteristics of the selected call and put option are recorded as of the date of entry and as of the day of expiration (e.g. delta on entry and delta on final trading day). Profitability of the call and put options in our strangle positions are estimated by taking the difference between the bid price on entry and the ask price on the day of expiration. We randomly select $75 \%$ of our sample for model training and $25 \%$ of our sample for 
out-of-sample testing. Furthermore, we rebalance our training data to have an equal number of profitable and losing strangles to avoid an imbalanced classes problem by resampling our training data to include more losses and less profits ${ }^{4}$. In total, the training data contains 19,430 positions and the test data contains 7,548 positions. All return calculations are annualized and made by assuming that four times the margin required to initiate the position is invested ${ }^{5}$. A more detailed discussion on returns, commissions and margin requirements is included in our appendix.

\section{Descriptive Statistics}

Our summary statistics are reported in Tables 1-5. As previously noted, the results show that shorting strangles across a variety of widths ${ }^{6}$ is generally profitable with mean returns ranging from about $8 \%$ to almost $11 \%$ on an annualized basis.

TABLE 1

RETURN BY WIDTH 60-60 DTE

\begin{tabular}{|c|c|c|c|c|c|c|}
\hline Width & Min. & 1st Qu. & Median & Mean & 3rd Qu. & Max. \\
\hline $1 \%$ & $-85.73 \%$ & $-6.22 \%$ & $9.15 \%$ & $7.73 \%$ & $21.22 \%$ & $138.60 \%$ \\
\hline $2 \%$ & $-87.74 \%$ & $-4.70 \%$ & $10.89 \%$ & $7.92 \%$ & $18.88 \%$ & $140.80 \%$ \\
\hline $3 \%$ & $-90.02 \%$ & $-0.76 \%$ & $10.37 \%$ & $8.62 \%$ & $17.65 \%$ & $140.50 \%$ \\
\hline $4 \%$ & $-91.92 \%$ & $4.23 \%$ & $9.38 \%$ & $8.93 \%$ & $16.54 \%$ & $139.80 \%$ \\
\hline $5 \%$ & $-93 \%$ & $4.33 \%$ & $8.18 \%$ & $8.87 \%$ & $14.84 \%$ & $140 \%$ \\
\hline $6 \%$ & $-93.92 \%$ & $4.15 \%$ & $7.67 \%$ & $9.20 \%$ & $14.31 \%$ & $131.40 \%$ \\
\hline $7 \%$ & $-95.08 \%$ & $4.51 \%$ & $7.84 \%$ & $9.89 \%$ & $14.48 \%$ & $132.10 \%$ \\
\hline $8 \%$ & $-96.12 \%$ & $4.72 \%$ & $7.93 \%$ & $10.10 \%$ & $15.11 \%$ & $139.60 \%$ \\
\hline $9 \%$ & $-97.13 \%$ & $4.51 \%$ & $7.71 \%$ & $10.45 \%$ & $15.02 \%$ & $141.60 \%$ \\
\hline $10 \%$ & $-97.96 \%$ & $4.37 \%$ & $7.66 \%$ & $10.64 \%$ & $14.87 \%$ & $144.20 \%$ \\
\hline $11 \%$ & $-98.04 \%$ & $3.93 \%$ & $6.95 \%$ & $10.02 \%$ & $13.52 \%$ & $146 \%$ \\
\hline $12 \%$ & $-97.92 \%$ & $3.63 \%$ & $6.59 \%$ & $9.42 \%$ & $12.60 \%$ & $135.80 \%$ \\
\hline $13 \%$ & $-97.80 \%$ & $3.38 \%$ & $6.37 \%$ & $8.81 \%$ & $12.08 \%$ & $128.20 \%$ \\
\hline $14 \%$ & $-97.61 \%$ & $3.55 \%$ & $6.41 \%$ & $9.13 \%$ & $11.82 \%$ & $113.80 \%$ \\
\hline $15 \%$ & $-97.36 \%$ & $3.59 \%$ & $6.95 \%$ & $9.71 \%$ & $12.39 \%$ & $108.70 \%$ \\
\hline
\end{tabular}

Note: Min. and Max. Returns on any individual position

However, the variability of returns on individual strangle positions, which we measure through maximum drawdowns, is alarmingly high. Drawdowns are measured for any given strangle position by tracking the differences between the bid price of the options at the time of entry and the prevailing asking prices until expiration. The maximum drawdown is the minimum of these differences.

We examine drawdowns of calls and puts separately and find that the put positions have on average larger maximum drawdowns as well as significantly larger worst case maximum drawdowns across all widths. The average maximum drawdowns of puts are about 12 percentage points greater than those of calls. We also note that the majority of the worst losses occurred in 2008. 
TABLE 2

CALL POSITIONS DRAWDOWN BY WIDTH 40-60 DTE

\begin{tabular}{|c|c|c|c|c|c|c|}
\hline Width & Min. & 1st Qu. & Median & Mean & 3rd Qu. & Max. \\
\hline $1 \%$ & $-80.47 \%$ & $-19.91 \%$ & $-10.25 \%$ & $-12.75 \%$ & $-3.22 \%$ & $12.80 \%$ \\
\hline $2 \%$ & $-82.52 \%$ & $-18.59 \%$ & $-8.16 \%$ & $-11.51 \%$ & $-2.01 \%$ & $12.78 \%$ \\
\hline $3 \%$ & $-86.24 \%$ & $-17.33 \%$ & $-6.02 \%$ & $-10.25 \%$ & $-1.28 \%$ & $12.40 \%$ \\
\hline $4 \%$ & $-84.42 \%$ & $-14.77 \%$ & $-3.89 \%$ & $-8.91 \%$ & $-0.77 \%$ & $12.00 \%$ \\
\hline $5 \%$ & $-86.43 \%$ & $-11.09 \%$ & $-2.20 \%$ & $-7.87 \%$ & $-0.41 \%$ & $10.67 \%$ \\
\hline $6 \%$ & $-90.07 \%$ & $-8.29 \%$ & $-1.46 \%$ & $-7.04 \%$ & $-0.27 \%$ & $11.65 \%$ \\
\hline $7 \%$ & $-82.49 \%$ & $-6.72 \%$ & $-1.04 \%$ & $-6.80 \%$ & $-0.19 \%$ & $10.97 \%$ \\
\hline $8 \%$ & $-91.08 \%$ & $-5.20 \%$ & $-0.79 \%$ & $-6.72 \%$ & $-0.14 \%$ & $10.62 \%$ \\
\hline $9 \%$ & $-84.38 \%$ & $-3.96 \%$ & $-0.57 \%$ & $-6.49 \%$ & $-0.13 \%$ & $10.52 \%$ \\
\hline $10 \%$ & $-86.37 \%$ & $-2.60 \%$ & $-0.47 \%$ & $-5.82 \%$ & $-0.14 \%$ & $10.72 \%$ \\
\hline $11 \%$ & $-94.30 \%$ & $-1.97 \%$ & $-0.41 \%$ & $-5.43 \%$ & $-0.13 \%$ & $10.46 \%$ \\
\hline $12 \%$ & $-78.86 \%$ & $-1.53 \%$ & $-0.38 \%$ & $-4.63 \%$ & $-0.15 \%$ & $10.22 \%$ \\
\hline $13 \%$ & $-93.16 \%$ & $-1.36 \%$ & $-0.38 \%$ & $-4.38 \%$ & $-0.15 \%$ & $9.81 \%$ \\
\hline $14 \%$ & $-90.46 \%$ & $-1.23 \%$ & $-0.39 \%$ & $-3.78 \%$ & $-0.15 \%$ & $9.18 \%$ \\
\hline $15 \%$ & $-72.69 \%$ & $-1.29 \%$ & $-0.44 \%$ & $-3.42 \%$ & $-0.16 \%$ & $8.69 \%$ \\
\hline
\end{tabular}

Note: Drawdown measured as 100(Min(bidentry - askt))/(4*Initial Margin)

TABLE 3

PUT POSITIONS DRAWDOWN BY WIDTH 40-60 DTE

\begin{tabular}{|c|c|c|c|c|c|c|}
\hline Width & Min. & 1st Qu. & Median & Mean & 3rd Qu. & Max. \\
\hline $1 \%$ & $-92.72 \%$ & $-17.09 \%$ & $-6.78 \%$ & $-12.16 \%$ & $-1.22 \%$ & $13.65 \%$ \\
\hline $2 \%$ & $-93.94 \%$ & $-16.60 \%$ & $-6.08 \%$ & $-11.87 \%$ & $-0.94 \%$ & $14.03 \%$ \\
\hline $3 \%$ & $-94.92 \%$ & $-16.98 \%$ & $-5.36 \%$ & $-11.36 \%$ & $-0.81 \%$ & $15.10 \%$ \\
\hline $4 \%$ & $-96.18 \%$ & $-17.56 \%$ & $-4.55 \%$ & $-11.08 \%$ & $-0.54 \%$ & $13.26 \%$ \\
\hline $5 \%$ & $-96.81 \%$ & $-17.29 \%$ & $-4.01 \%$ & $-10.72 \%$ & $-0.53 \%$ & $13.84 \%$ \\
\hline $6 \%$ & $-97.34 \%$ & $-16.58 \%$ & $-3.66 \%$ & $-10.64 \%$ & $-0.43 \%$ & $16.46 \%$ \\
\hline $7 \%$ & $-97.90 \%$ & $-18.40 \%$ & $-3.86 \%$ & $-11.16 \%$ & $-0.39 \%$ & $16.69 \%$ \\
\hline $8 \%$ & $-98.61 \%$ & $-16.80 \%$ & $-3.34 \%$ & $-11.07 \%$ & $-0.21 \%$ & $17.79 \%$ \\
\hline $9 \%$ & $-98.93 \%$ & $-17.44 \%$ & $-3.24 \%$ & $-11.47 \%$ & $-0.21 \%$ & $14.84 \%$ \\
\hline $10 \%$ & $-99.22 \%$ & $-15.49 \%$ & $-2.82 \%$ & $-11.18 \%$ & $-0.10 \%$ & $16.23 \%$ \\
\hline $11 \%$ & $-99.07 \%$ & $-14.35 \%$ & $-2.44 \%$ & $-10.94 \%$ & $-0.10 \%$ & $19.84 \%$ \\
\hline $12 \%$ & $-98.95 \%$ & $-12.54 \%$ & $-2.44 \%$ & $-10.58 \%$ & $-0.06 \%$ & $20.28 \%$ \\
\hline $13 \%$ & $-98.88 \%$ & $-10.53 \%$ & $-2.01 \%$ & $-9.92 \%$ & $-0.08 \%$ & $18.66 \%$ \\
\hline $14 \%$ & $-98.75 \%$ & $-8.39 \%$ & $-1.86 \%$ & $-9.23 \%$ & $0 \%$ & $15.21 \%$ \\
\hline $15 \%$ & $-98.60 \%$ & $-7.53 \%$ & $-1.68 \%$ & $-8.55 \%$ & $0 \%$ & $18.81 \%$ \\
\hline
\end{tabular}

Note: Drawdown measured as 100(Min(bidentry - askt))/(4*Initial Margin)

As reported in Table 4, average returns were positive in each year. Interestingly, the positions generated a remarkably high average return of $9.87 \%$ during the crisis year 2008 . The same year also happens to have had the largest loss on any one position of over $98 \%$. The fact that the highest average 
return was achieved in 2009 suggests that option prices had a long memory of fear embedded, resulting in relatively expensive option prices which subsequently made the selling of options an attractive proposition.

TABLE 4

ANNUALIZED RETURN BY YEAR 40-60 DTE

\begin{tabular}{|c|c|c|c|c|c|c|c|c|}
\hline \hline Year & N & Min. & 1st Qu. & Median & Mean & 3rd Qu. & Max. \\
\hline 2005 & 1085 & $-31.22 \%$ & $2.91 \%$ & $5.70 \%$ & $5.02 \%$ & $9.99 \%$ & $25.33 \%$ \\
\hline 2006 & 1117 & $-24.31 \%$ & $2.05 \%$ & $5.02 \%$ & $4.31 \%$ & $9.08 \%$ & $29.55 \%$ \\
\hline 2007 & 1564 & $-53.81 \%$ & $4.08 \%$ & $11.14 \%$ & $9.95 \%$ & $20.67 \%$ & $56.49 \%$ \\
\hline 2008 & 2405 & $-98.04 \%$ & $-15.87 \%$ & $13.47 \%$ & $9.87 \%$ & $34.32 \%$ & $146.00 \%$ \\
\hline 2009 & 2501 & $-89.84 \%$ & $9.31 \%$ & $20.46 \%$ & $18.28 \%$ & $33.77 \%$ & $90.83 \%$ \\
\hline 2010 & 2791 & $-51.23 \%$ & $4.93 \%$ & $11.43 \%$ & $11.44 \%$ & $20.35 \%$ & $76.79 \%$ \\
\hline 2011 & 2763 & $-80.76 \%$ & $6.99 \%$ & $16.69 \%$ & $16.85 \%$ & $32.59 \%$ & $88.03 \%$ \\
\hline 2012 & 2780 & $-36.32 \%$ & $4.59 \%$ & $8.35 \%$ & $8.44 \%$ & $13.78 \%$ & $43.57 \%$ \\
\hline 2013 & 2151 & $-35.44 \%$ & $3.41 \%$ & $5.75 \%$ & $5.54 \%$ & $9.46 \%$ & $28.79 \%$ \\
\hline 2014 & 1729 & $-51.09 \%$ & $4.32 \%$ & $7.10 \%$ & $6.94 \%$ & $10.98 \%$ & $41.55 \%$ \\
\hline 2015 & 2324 & $-52.15 \%$ & $4.16 \%$ & $8.20 \%$ & $6.03 \%$ & $13.53 \%$ & $42.88 \%$ \\
\hline 2016 & 2525 & $-33.19 \%$ & $4.25 \%$ & $7.42 \%$ & $7.74 \%$ & $11.83 \%$ & $48.42 \%$ \\
\hline 2017 & 2264 & $-44.00 \%$ & $2.38 \%$ & $3.84 \%$ & $2.99 \%$ & $6.10 \%$ & $19.19 \%$ \\
\hline 2018 & 2186 & $-22.96 \%$ & $3.28 \%$ & $5.84 \%$ & $7.09 \%$ & $9.71 \%$ & $47.09 \%$ \\
\hline
\end{tabular}

Note: Summary statistics aggregated across all individual positions.

Table 5 shows a more detailed analysis of the returns by year and by width. It is equally remarkable to see that even during the height of the financial crisis in 2008, average returns across all widths were positive. More surprisingly, even the $1 \%$ strangle width position had a positive average return of over $9 \%$, which seems counter-intuitive at first. On second thought, however, it shows that the much larger premium income must have been a significant compensating factor in balancing the higher risk from options expiring in-the-money. Our detailed results in Table 5 also suggest that there may not be a "sweet spot" in terms of identifying an optimal width for our strategy. The maximum average return in each year is not consistent across strangle widths. We find that maximum average returns were achieved at the $1 \%$ width in years 2005, 2011, 2014 and 2016 and at a 10\% width during the years 2007, 2009, and 2010. The overall largest average return of over $23 \%$ occurred in 2009 at a width of $10 \%$ further evidence of a long memory of fear in embedded option prices. 
TABLE 5

AVERAGE ANNUALIZED RETURN BY YEAR AND WIDTH 40-60 DTE

\begin{tabular}{|c|c|c|c|c|c|c|c|c|c|c|c|c|c|c|c|}
\hline Year & $1 \%$ & $2 \%$ & $3 \%$ & $4 \%$ & $5 \%$ & $6 \%$ & $7 \%$ & $8 \%$ & $9 \%$ & $10 \%$ & $11 \%$ & $12 \%$ & $13 \%$ & $14 \%$ & $15 \%$ \\
\hline 2005 & $6.06 \% * *$ & $5.82 \%$ & $5.24 \%$ & $4.77 \%$ & $4.50 \%$ & $4.09 \%$ & $4.26 \%$ & $4.40 \%$ & $4.07 \%$ & $3.80 \%$ & $3.33 \%$ & - & - & - & - \\
\hline 2006 & $2.46 \%$ & $4.30 \%$ & $4.83 \%$ & $5.07 \%$ & $4.92 \%$ & $4.23 \%$ & $4.60 \%$ & $5.24 \%$ & $6.28 \% * *$ & $6.15 \%$ & - & - & - & - & - \\
\hline 2007 & $8.30 \%$ & $8.57 \%$ & $8.82 \%$ & $9.14 \%$ & $9.71 \%$ & $10.17 \%$ & $11.06 \%$ & $12.31 \%$ & $12.56 \%$ & $13.34 \% * *$ & $12.98 \%$ & $11.16 \%$ & $10.41 \%$ & $9.59 \%$ & $8.59 \%$ \\
\hline 2008 & $9.25 \%$ & $9.39 \%$ & $9.84 \%$ & $10.59 \%$ & $9.38 \%$ & $9.50 \%$ & $12.35 \% * *$ & $10.39 \%$ & $11.54 \%$ & $10.52 \%$ & $10.36 \%$ & $8.63 \%$ & $6.57 \%$ & $8.84 \%$ & $10.01 \%$ \\
\hline 2009 & $14.17 \%$ & $15.63 \%$ & $18.92 \%$ & $18.59 \%$ & $18.62 \%$ & $19.64 \%$ & $19.16 \%$ & $18.64 \%$ & $21.54 \%$ & $23.08 \% * *$ & $20.02 \%$ & $18.67 \%$ & $16.14 \%$ & $15.22 \%$ & $16.19 \%$ \\
\hline 2010 & $5.25 \%$ & $6.25 \%$ & $7.63 \%$ & $9.23 \%$ & $10.93 \%$ & $12.61 \%$ & $14.17 \%$ & $14.97 \%$ & $15.31 \%$ & $15.59 \% * *$ & $14.58 \%$ & $.03 \%$ & $1.57 \%$ & $0.30 \%$ & $9.43 \%$ \\
\hline 2011 & $18.64 \% * *$ & $18.39 \%$ & $17.80 \%$ & $17.39 \%$ & $17.10 \%$ & $16.84 \%$ & $16.55 \%$ & $16.20 \%$ & $16.53 \%$ & $17.07 \%$ & $16.98 \%$ & $15.89 \%$ & $15.60 \%$ & $15.35 \%$ & $14.72 \%$ \\
\hline 2012 & $6.79 \%$ & $7.48 \%$ & $8.16 \%$ & $9.12 \%$ & $9.86 \%$ & $10.69 \%$ & $11.28 \% * *$ & $10.74 \%$ & $9.84 \%$ & $9.02 \%$ & $7.59 \%$ & $6.50 \%$ & $5.83 \%$ & $5.26 \%$ & $5.01 \%$ \\
\hline 2013 & $5.27 \%$ & $5.66 \%$ & $6.21 \%$ & $6.55 \%$ & $6.67 \%$ & $6.68 \% * *$ & $6.49 \%$ & $5.94 \%$ & $5.20 \%$ & $4.73 \%$ & $3.97 \%$ & $3.39 \%$ & $2.94 \%$ & $2.62 \%$ & $2.14 \%$ \\
\hline 2014 & $9.45 \% * *$ & $7.42 \%$ & $8.21 \%$ & $8.34 \%$ & $7.01 \%$ & $6.38 \%$ & $6.35 \%$ & $5.75 \%$ & $5.19 \%$ & $4.98 \%$ & $4.85 \%$ & $4.06 \%$ & $3.36 \%$ & $3.56 \%$ & $2.80 \%$ \\
\hline 2015 & $4.55 \%$ & $4.06 \%$ & $4.85 \%$ & $4.92 \%$ & $5.19 \%$ & $5.99 \%$ & $6.41 \%$ & $8.33 \% * *$ & $8.17 \%$ & $8.08 \%$ & $7.62 \%$ & $7.30 \%$ & $6.67 \%$ & $6.24 \%$ & $7.20 \%$ \\
\hline 2016 & $9.72 \% * *$ & $9.11 \%$ & $9.33 \%$ & $9.11 \%$ & $8.43 \%$ & $7.88 \%$ & $7.39 \%$ & $7.28 \%$ & $7.12 \%$ & $7.22 \%$ & $6.29 \%$ & $5.60 \%$ & $4.89 \%$ & $4.92 \%$ & $3.72 \%$ \\
\hline 2017 & $1.59 \%$ & $2.30 \%$ & $3.13 \%$ & $3.71 \%$ & $3.92 \% * *$ & $3.66 \%$ & $3.49 \%$ & $3.51 \%$ & $3.26 \%$ & $2.95 \%$ & $2.50 \%$ & $2.18 \%$ & $1.93 \%$ & $1.72 \%$ & $1.67 \%$ \\
\hline 2018 & $7.98 \%$ & $8.69 \%$ & $9.68 \%$ ** & $9.57 \%$ & $8.86 \%$ & $7.97 \%$ & $7.16 \%$ & $6.35 \%$ & $6.09 \%$ & $5.62 \%$ & $5.21 \%$ & $4.65 \%$ & $4.65 \%$ & $3.89 \%$ & $4.02 \%$ \\
\hline
\end{tabular}

Note: Maximum denoted by $* *$ in each row.

\section{Results}

Across each of our estimation methods we sequentially add in explanatory variables across 8 specifications and multiply all of our dependent variables, with the exception of premiums collected, by 100 (i.e. Delta of 0.4 becomes 40, allowing for easier interpretation and a cleaner scaling of the average partial effects). We begin by first including simple terms that characterize the option contracts which include implied volatility and the prevailing bid price of the call and put option that form a strangle. Later, we sequentially add in comparative statics, or greeks, from the BSM framework one at a time in order to determine the relative importance of various sensitivities to strangle profitability. In our final two specifications we include controls for the Width and Width ${ }^{2}$ of each strangle. The quadratic term is included in the final specification in order to determine the optimal strangle width to have the highest average likelihood of profitability. Throughout each of our specifications, we will be reducing omitted variable bias, which will allow us to determine which variables have substantial influence over the likelihood of profitability of a strangle position. White (1980) heteroskedasticity robust standard errors are reported for our linear probability model estimates in Table 6. 


\section{TABLE 6 \\ OLS: LIMEAR PROBABILITY MODEL FOR STRANGLE PROFIT 40-60 DTE}

\begin{tabular}{|c|c|c|c|c|c|c|c|c|}
\hline \multicolumn{9}{|c|}{ Dependent variable: } \\
\hline \multicolumn{9}{|c|}{ I(ifelse(strangle_pl > profitability_limit, 1,0)) } \\
\hline & (1) & (2) & (3) & (4) & $(5)$ & (6) & (7) & (8) \\
\hline \multirow[t]{2}{*}{ Call Entry IV } & $-0.016^{* * *}$ & -0.0003 & $-0.003^{* *}$ & $-0.006 * * *$ & 0.001 & $0.005^{* *}$ & $0.008^{* * *}$ & $0.011^{* * *}$ \\
\hline & $(0.002)$ & $(0.001)$ & $(0.001)$ & $(0.001)$ & $(0.002)$ & $(0.002)$ & $(0.002)$ & $(0.002)$ \\
\hline \multirow[t]{2}{*}{ Call Premium Collected } & $-0.101^{* * *}$ & $0.089 * * *$ & $-0.212 * * *$ & $-0.248 * * *$ & $-0.211 * * *$ & $-0.225^{* * *}$ & $-0.182 * * *$ & $-0.328 * * *$ \\
\hline & $(0.009)$ & $(0.014)$ & $(0.020)$ & $(0.020)$ & $(0.023)$ & $(0.024)$ & $(0.024)$ & $(0.026)$ \\
\hline \multirow[t]{2}{*}{ Call Entry Delta } & & $-0.015^{* * *}$ & $0.017^{* * *}$ & $0.018^{* * *}$ & $0.022^{* * *}$ & $0.020 * * *$ & $0.016^{* * *}$ & $0.012^{* * *}$ \\
\hline & & $(0.001)$ & $(0.002)$ & $(0.002)$ & $(0.002)$ & $(0.003)$ & $(0.003)$ & $(0.003)$ \\
\hline \multirow[t]{2}{*}{ Call Entry Gamma } & & & $-0.210^{* * *}$ & $-0.205^{* * *}$ & $-0.183^{* * *}$ & $-0.203^{* * *}$ & $-0.170 * * *$ & $-0.174 * * *$ \\
\hline & & & $(0.010)$ & $(0.011)$ & $(0.011)$ & $(0.014)$ & $(0.015)$ & $(0.015)$ \\
\hline \multirow[t]{2}{*}{ Call Entry Vega } & & & & 0.001 & $0.006^{* * *}$ & $0.014^{* * *}$ & $0.011^{* *}$ & -0.0003 \\
\hline & & & & $(0.002)$ & $(0.002)$ & $(0.005)$ & $(0.005)$ & $(0.005)$ \\
\hline \multirow[t]{2}{*}{ Call Entry Theta } & & & & & $0.101^{* * *}$ & $0.155^{* * *}$ & $0.152^{* * *}$ & $0.118^{* * *}$ \\
\hline & & & & & $(0.011)$ & $(0.014)$ & $(0.014)$ & $(0.015)$ \\
\hline \multirow[t]{2}{*}{ Call Entry Rho } & & & & & & 0.013 & 0.006 & $0.046 * * *$ \\
\hline & & & & & & $(0.013)$ & $(0.013)$ & $(0.013)$ \\
\hline \multirow{2}{*}{ Put Entry IV } & $0.021^{* * *}$ & 0.001 & $0.002^{* *}$ & -0.0002 & -0.001 & $-0.003^{*}$ & $-0.011^{* * *}$ & $-0.005^{* *}$ \\
\hline & $(0.001)$ & $(0.001)$ & $(0.001)$ & $(0.001)$ & $(0.001)$ & $(0.002)$ & $(0.002)$ & $(0.002)$ \\
\hline \multirow[t]{2}{*}{ Put Premium Collected } & $-0.025 * * *$ & 0.012 & $0.286 * * *$ & $0.353^{* * *}$ & $0.388 * * *$ & $0.456 * * *$ & $0.467 * * *$ & $0.531^{* * *}$ \\
\hline & $(0.007)$ & $(0.011)$ & $(0.019)$ & $(0.022)$ & $(0.025)$ & $(0.027)$ & $(0.027)$ & $(0.027)$ \\
\hline \multirow[t]{2}{*}{ Put Entry Delta } & & $0.009 * * *$ & $0.044^{* * *}$ & $0.045^{* * *}$ & $0.053^{* * *}$ & $0.047^{* * *}$ & $0.040 * * *$ & $0.047^{* * *}$ \\
\hline & & $(0.001)$ & $(0.002)$ & $(0.002)$ & $(0.003)$ & $(0.003)$ & $(0.003)$ & $(0.003)$ \\
\hline \multirow[t]{2}{*}{ Put Entry Gamma } & & & $0.285^{* * *}$ & $0.276^{* * *}$ & $0.265^{* * *}$ & $0.272^{* * *}$ & $0.252^{* * *}$ & $0.235^{* * *}$ \\
\hline & & & $(0.015)$ & $(0.016)$ & $(0.016)$ & $(0.018)$ & $(0.019)$ & $(0.019)$ \\
\hline \multirow[t]{2}{*}{ Put Entry Vega } & & & & $-0.008^{* * *}$ & $-0.007^{* * *}$ & 0.002 & $0.011^{* * *}$ & 0.003 \\
\hline & & & & $(0.002)$ & $(0.002)$ & $(0.002)$ & $(0.003)$ & $(0.003)$ \\
\hline \multirow[t]{2}{*}{ Put Entry Theta } & & & & & -0.007 & 0.003 & 0.005 & 0.011 \\
\hline & & & & & $(0.009)$ & $(0.009)$ & $(0.009)$ & $(0.009)$ \\
\hline \multirow[t]{2}{*}{ Put Entry Rho } & & & & & & $0.063^{* * *}$ & $0.080^{* * *}$ & $0.077^{* * *}$ \\
\hline & & & & & & $(0.009)$ & $(0.009)$ & $(0.009)$ \\
\hline \multirow[t]{2}{*}{ Width } & & & & & & & $0.027^{* * *}$ & $-0.094 * * *$ \\
\hline & & & & & & & $(0.004)$ & $(0.011)$ \\
\hline \multirow[t]{2}{*}{ I(Width^2) } & & & & & & & & $0.005^{* * *}$ \\
\hline & & & & & & & & $(0.000)$ \\
\hline \multirow[t]{2}{*}{ Constant } & $0.439 * * *$ & $0.860 * * *$ & $0.791^{* * *}$ & $0.948^{* * *}$ & $0.926 * * *$ & $0.890^{* * *}$ & $0.656^{* * *}$ & $1.341^{* * *}$ \\
\hline & $(0.010)$ & $(0.015)$ & $(0.016)$ & $(0.026)$ & $(0.026)$ & $(0.027)$ & $(0.043)$ & $(0.071)$ \\
\hline Observations & 22,636 & 22,636 & 22,636 & 22,636 & 22,636 & 22,636 & 22,636 & 22,636 \\
\hline R2 & 0.139 & 0.19 & 0.206 & 0.208 & 0.213 & 0.215 & 0.216 & 0.222 \\
\hline Adjusted R2 & 0.139 & 0.19 & 0.206 & 0.208 & 0.212 & 0.214 & 0.216 & 0.221 \\
\hline Residual Std. Error & $0.464(d f=22631)$ & $0.450(d f=22629)$ & $0.446(d f=22627)$ & $0.445(d f=22625)$ & $0.444(d f=22623)$ & $0.443(\mathrm{df}=22621)$ & $0.443(d f=22620)$ & $0.441(\mathrm{df}=22619)$ \\
\hline F Statistic & $\begin{array}{c}910.940 * * * \\
(\mathrm{df}=4 ; 22631)\end{array}$ & $\begin{array}{c}885.866^{* * *} \\
(\mathrm{df}=6 ; 22629)\end{array}$ & $\begin{array}{c}735.486^{* * *} \\
(\mathrm{df}=8 ; 22627)\end{array}$ & $\begin{array}{c}594.801 * * * \\
(\mathrm{df}=10 ; 22625)\end{array}$ & $\begin{array}{c}509.743^{* * *} \\
(\mathrm{df}=12 ; 22623)\end{array}$ & $\begin{array}{c}441.954^{* * *} \\
(\mathrm{df}=14 ; 22621)\end{array}$ & $\begin{array}{c}416.154^{* * *} \\
(\mathrm{df}=15 ; 22620)\end{array}$ & $\begin{array}{c}402.855 * * * \\
(\mathrm{df}=16 ; 22619)\end{array}$ \\
\hline
\end{tabular}

Results of our logistic regressions are reported in Table 7. 
TABLE 7

LOGISTIC REGRESSION FOR STRANGLE PROFIT 40-60 DTE

\begin{tabular}{|c|c|c|c|c|c|c|c|c|}
\hline \multicolumn{9}{|c|}{$\begin{array}{c}\text { Dependent variable: } \\
\text { Strangle Profit }>0\end{array}$} \\
\hline & (1) & $(2)$ & (3) & (4) & (5) & $(6)$ & (7) & $(8)$ \\
\hline \multirow[t]{2}{*}{ Call Entry IV } & $-0.071 * * *$ & 0.0003 & $-0.017 * *$ & $-0.029 * * *$ & 0.002 & 0.018 & $0.034^{* * *}$ & $0.048 * * *$ \\
\hline & $(0.008)$ & $(0.008)$ & $(0.008)$ & $(0.008)$ & $(0.009)$ & $(0.013)$ & $(0.013)$ & $(0.013)$ \\
\hline \multirow[t]{2}{*}{ Call Premium Collected } & $-0.495 * * *$ & $0.381 * * *$ & $-0.956 * * *$ & $-1.145^{* * *}$ & $-0.920 * * *$ & $-0.974 * * *$ & $-0.703^{* * *}$ & $-1.469 * * *$ \\
\hline & $(0.045)$ & $(0.069)$ & $(0.100)$ & $(0.105)$ & $(0.117)$ & $(0.125)$ & $(0.130)$ & $(0.146)$ \\
\hline \multirow[t]{2}{*}{ Call Entry Delta } & & $-0.065 * * *$ & $0.078 * * *$ & $0.085^{* * *}$ & $0.107^{* * *}$ & $0.102 * * *$ & $0.086^{* * *}$ & $0.066 * * *$ \\
\hline & & $(0.006)$ & (0.009) & $(0.010)$ & $(0.010)$ & $(0.014)$ & $(0.014)$ & $(0.014)$ \\
\hline \multirow[t]{2}{*}{ Call Entry Gamma } & & & $-0.964 * * *$ & $-0.942 * * *$ & $-0.824 * * *$ & $-0.938 * * *$ & $-0.739 * * *$ & $-0.767 * * *$ \\
\hline & & & $(0.050)$ & $(0.055)$ & $(0.056)$ & $(0.071)$ & $(0.075)$ & $(0.076)$ \\
\hline \multirow[t]{2}{*}{ Call Entry Vega } & & & & 0.003 & $0.033^{* * *}$ & $0.080 * * *$ & $0.067 * * *$ & 0.016 \\
\hline & & & & $(0.009)$ & $(0.010)$ & $(0.024)$ & $(0.025)$ & $(0.025)$ \\
\hline \multirow[t]{2}{*}{ Call Entry Theta } & & & & & $0.553^{* * *}$ & $0.811^{* * *}$ & $0.810 * * *$ & $0.644 * * *$ \\
\hline & & & & & $(0.056)$ & $(0.073)$ & $(0.074)$ & $(0.075)$ \\
\hline \multirow[t]{2}{*}{ Call Entry Rho } & & & & & & 0.033 & -0.024 & $0.169 * *$ \\
\hline & & & & & & $(0.065)$ & $(0.066)$ & $(0.068)$ \\
\hline \multirow[t]{2}{*}{ Put Entry IV } & $0.093 * * *$ & 0.003 & $0.012^{*}$ & -0.002 & -0.004 & -0.011 & $-0.061^{* * *}$ & $-0.028 * *$ \\
\hline & $(0.006)$ & $(0.006)$ & $(0.006)$ & $(0.007)$ & $(0.007)$ & $(0.009)$ & $(0.012)$ & $(0.012)$ \\
\hline \multirow[t]{2}{*}{ Put Premium Collected } & $-0.093^{* * *}$ & $0.110^{*}$ & $1.313^{* * *}$ & $1.651^{* * *}$ & $1.778^{* * *}$ & $2.095^{* * *}$ & $2.169 * * *$ & $2.533^{* * *}$ \\
\hline & $(0.034)$ & $(0.057)$ & $(0.095)$ & $(0.107)$ & $(0.122)$ & $(0.132)$ & $(0.132)$ & $(0.137)$ \\
\hline \multirow[t]{2}{*}{ Put Entry Delta } & & $0.045^{* * *}$ & $0.205^{* * *}$ & $0.211^{* * *}$ & $0.252^{* * *}$ & $0.230 * * *$ & $0.186^{* * *}$ & $0.223^{* * *}$ \\
\hline & & $(0.007)$ & $(0.012)$ & $(0.012)$ & $(0.013)$ & $(0.015)$ & $(0.016)$ & $(0.016)$ \\
\hline \multirow[t]{2}{*}{ Put Entry Gamma } & & & $1.310 * * *$ & $1.259 * * *$ & $1.192 * * *$ & $1.256^{* * *}$ & $1.122^{* * *}$ & $1.044^{* * *}$ \\
\hline & & & $(0.073)$ & $(0.079)$ & $(0.080)$ & $(0.090)$ & (0.091) & $(0.092)$ \\
\hline \multirow[t]{2}{*}{ Put Entry Vega } & & & & $-0.040 * * *$ & $-0.039 * * *$ & 0.0005 & $0.053^{* * *}$ & 0.013 \\
\hline & & & & $(0.008)$ & (0.009) & $(0.012)$ & $(0.014)$ & $(0.014)$ \\
\hline \multirow[t]{2}{*}{ Put Entry Theta } & & & & & $-0.073^{*}$ & -0.025 & -0.014 & 0.029 \\
\hline & & & & & $(0.044)$ & $(0.045)$ & $(0.045)$ & $(0.045)$ \\
\hline \multirow[t]{2}{*}{ Put Entry Rho } & & & & & & $0.281 * * *$ & $0.387^{* * *}$ & $0.376 * * *$ \\
\hline & & & & & & $(0.045)$ & $(0.048)$ & $(0.047)$ \\
\hline \multirow[t]{2}{*}{ Width } & & & & & & & $0.168^{* * *}$ & $-0.470 * * *$ \\
\hline & & & & & & & $(0.023)$ & $(0.054)$ \\
\hline \multirow[t]{2}{*}{ I(Width^2) } & & & & & & & & $0.026 * * *$ \\
\hline & & & & & & & & $(0.002)$ \\
\hline \multirow[t]{2}{*}{ Constant } & $-0.261 * * *$ & $1.681^{* * *}$ & $1.378^{* * *}$ & $2.191^{* * *}$ & $2.088^{* * *}$ & $1.902 * * *$ & $0.505^{* *}$ & $4.084 * * *$ \\
\hline & $(0.047)$ & $(0.084)$ & $(0.090)$ & $(0.144)$ & $(0.145)$ & $(0.147)$ & $(0.239)$ & $(0.365)$ \\
\hline Pseduo-R2 & 0.107 & 0.146 & 0.159 & 0.16 & 0.165 & 0.166 & 0.168 & 0.173 \\
\hline Observations & 22,636 & 22,636 & 22,636 & 22,636 & 22,636 & 22,636 & 22,636 & 22,636 \\
\hline Log Likelihood & $-14,008.56$ & $-13,394.52$ & $-13,203.09$ & $-13,176.03$ & $-13,106.69$ & $-13,084.05$ & $-13,056.62$ & $-12,969.53$ \\
\hline Akaike Inf. Crit. & $28,027.12$ & $26,803.04$ & $26,424.18$ & $26,374.06$ & $26,239.38$ & $26,198.10$ & $26,145.24$ & $25,973.06$ \\
\hline
\end{tabular}

Note: $* \mathrm{p}<0.1 ; * * \mathrm{p}<0.05 ; * * * \mathrm{p}<0.01$

Pseduo- $\mathrm{R}^{2}$ is calculated for each of our specifications by computing 1 - Model Deviance/Null Deviance.

A priori, the expected sign on call premium, put premium, call implied volatility, and put implied volatility are all ambiguous since an increase in implied volatility corresponds to a higher option price. The higher option price provides the seller of the option a larger cushion against losses but the higher implied volatility suggests that there is an increased likelihood that a large move may occur in the underlying security. The estimates of our linear probability model and logistic regression specifications seem to confirm our priors with sign changes across our specifications. 
The average partial effects, shown in Figure 2, are derived from our fully specified logistic regression, in which implied volatility has a near zero effect. Interestingly, call and put premiums at the time of entry have large and economically significant signs that are opposite from one another, indicating that the larger (smaller) the put (call) premium collected the greater (lower) the likelihood of profitability. The increasing likelihood of profitability associated with an increase in put premium collected poses a challenge for our model, which will leave the model exposed to the potential for large drawdowns, as the higher premium is associated with increased risk.

Delta and Gamma represent the first and second partial derivatives of the BSM framework with respect to the underlying price. In theory, as Delta and Gamma increase in absolute value, the likelihood of the underlying security going into the money increases, therefore an option seller is negatively impacted by a rise in Delta or Gamma. A low Delta on calls is associated with a low probability of the option ending in the money, while for puts a high Delta is associated with a low probability of the option ending in the money. Our results in both model estimation techniques indicate that a high put Delta is more impactful on predicting strangle profitability than a call's Delta, where a one percentage point increase in Delta for puts increases strangle profitability by $2.23 \%$ and a one percentage point increase in Delta for calls increases strangle profitability by $0.6 \%$.

Vega captures the sensitivity of the option price with respect to changes in the volatility of the underlying security. As Vega increases, options prices begin to move faster in response to changes in volatility. From the perspective of an option seller, the faster changes of the options prices correspond to an increase in the risk of the option going into the money. Faster changes of the options prices can be both dangerous and helpful for a strangle position leaving an ambiguous effect on option strangles, which is captured by the statistical insignificance of Vega on predicting strangle profitability in our fully specified models.

Rho represents the sensitivity of the option price with respect to changes in the risk-free rate of interest. Generally, call options prices increase as the risk-free rate of interest rises and put options prices decrease as the risk-free rate of interest rises. Our estimated models indicate that an increase in Rho for puts in our strangle positions increases the likelihood of strangle profitability, in-line with theoretical predictions.

Lastly, Theta represents the sensitivity of the option price with respect to time. As discussed previously, the likelihood of an option expiring in the money diminishes exponentially as the option approaches expiration. As a result, a strangle position should have an increasing likelihood of profitability as Theta increases in absolute terms. Our empirical results suggest that we cannot reject the hypothesis that an increase in Theta leads to a higher likelihood of strangle profitability by the significant and positive coefficients on call Theta. 
TABLE 8

EXAMPLE PREDICTIONS OF MODELS

\begin{tabular}{|c|c|c|c|c|c|c|c|c|c|c|c|c|c|c|c|c|}
\hline \multirow[b]{2}{*}{ Width } & \multicolumn{7}{|c|}{ Call } & \multicolumn{7}{|c|}{ Put } & \multirow{2}{*}{$\begin{array}{l}\text { Prob. } \\
\text { LPM }\end{array}$} & \multirow{2}{*}{$\begin{array}{l}\text { Prob. } \\
\text { Logistic }\end{array}$} \\
\hline & Entry IV & $\begin{array}{l}\text { Premium } \\
\text { Collected }\end{array}$ & $\begin{array}{l}\text { Entry } \\
\text { Delta }\end{array}$ & $\begin{array}{c}\text { Entry } \\
\text { Gamma }\end{array}$ & $\begin{array}{l}\text { Entry } \\
\text { Vega }\end{array}$ & $\begin{array}{l}\text { Entry } \\
\text { Theta }\end{array}$ & Entry Rho & Entry IV & $\begin{array}{l}\text { Premium } \\
\text { Collected } \\
\end{array}$ & $\begin{array}{l}\text { Entry } \\
\text { Delta }\end{array}$ & $\begin{array}{c}\text { Entry } \\
\text { Gamma }\end{array}$ & $\begin{array}{l}\text { Entry } \\
\text { Vega }\end{array}$ & $\begin{array}{l}\text { Entry } \\
\text { Theta }\end{array}$ & Entry Rho & & \\
\hline $1 \%$ & 15.525 & 2.519 & 41.087 & 23.266 & 4.787 & -3.164 & 8.796 & 16.968 & 2.999 & -40.967 & 23.525 & 4.351 & -3.847 & -8.99 & 0.239 & 0 \\
\hline $2 \%$ & 14.667 & 1.804 & 32.68 & 21.253 & 4.564 & -2.807 & 6.938 & 17.537 & 2.538 & -34.975 & 22.595 & 3.887 & -3.857 & -7.759 & 0.294 & 0 \\
\hline $3 \%$ & 14.957 & 1.384 & 26.476 & 18.114 & 4.153 & -2.509 & 5.337 & 19.135 & 2.225 & -30.253 & 20.557 & 3.454 & -3.784 & -6.516 & 0.358 & 0 \\
\hline $4 \%$ & 14.914 & 1.041 & 20.838 & 14.915 & 3.55 & -2.124 & 4.031 & 20.175 & 1.94 & -26.301 & 18.727 & 3.05 & -3.657 & -5.599 & 0.432 & 0 \\
\hline $5 \%$ & 14.527 & 0.751 & 16.026 & 12.128 & 2.971 & -1.749 & 3.024 & 20.851 & 1.651 & -22.648 & 17.103 & 2.69 & -3.457 & -4.819 & 0.499 & 0 \\
\hline $6 \%$ & 15.263 & 0.63 & 13.285 & 10.144 & 2.452 & -1.591 & 2.396 & 22.466 & 1.542 & -20.382 & 15.739 & 2.368 & -3.461 & -4.293 & 0.553 & 0.218 \\
\hline $7 \%$ & 15.592 & 0.525 & 10.678 & 8.331 & 1.937 & -1.411 & 1.88 & 23.686 & 1.405 & -17.965 & 14.474 & 2.038 & -3.373 & -3.782 & 0.608 & 0.477 \\
\hline $8 \%$ & 15.984 & 0.433 & 8.983 & 7.02 & 1.657 & -1.247 & 1.515 & 25.057 & 1.291 & -16.318 & 13.429 & 1.828 & -3.304 & -3.396 & 0.639 & 0.634 \\
\hline $9 \%$ & 16.514 & 0.364 & 7.466 & 5.78 & 1.365 & -1.114 & 1.201 & 26.145 & 1.141 & -14.209 & 11.959 & 1.605 & -3.126 & -2.904 & 0.685 & 0.872 \\
\hline $10 \%$ & 16.328 & 0.271 & 5.933 & 4.899 & 1.121 & -0.95 & 0.976 & 26.482 & 0.993 & -12.438 & 11.009 & 1.415 & -2.919 & -2.591 & 0.725 & 1 \\
\hline $11 \%$ & 16.963 & 0.225 & 5.363 & 4.526 & 1.003 & -0.901 & 0.889 & 27.383 & 0.892 & -11.146 & 9.902 & 1.281 & -2.71 & -2.291 & 0.762 & 1 \\
\hline $12 \%$ & 17.842 & 0.19 & 4.932 & 4.404 & 0.895 & -0.916 & 0.846 & 28.597 & 0.832 & -10.19 & 9.21 & 1.153 & -2.657 & -2.095 & 0.799 & 1 \\
\hline $13 \%$ & 18.587 & 0.15 & 4.489 & 4.142 & 0.818 & -0.882 & 0.777 & 29.529 & 0.749 & -9.234 & 8.413 & 1.053 & -2.499 & -1.875 & 0.843 & 1 \\
\hline $14 \%$ & 20.574 & 0.209 & 5.198 & 4.196 & 0.85 & -1.03 & 0.822 & 32.354 & 0.797 & -9.325 & 7.837 & 1.028 & -2.585 & -1.761 & 0.875 & 1 \\
\hline $15 \%$ & 21.994 & 0.209 & 5.375 & 4.629 & 0.838 & -1.161 & 0.9 & 34.459 & 0.814 & -9.116 & 7.597 & 0.962 & -2.63 & -1.706 & 0.924 & 1 \\
\hline
\end{tabular}

Note: Each of the examples is the mean of each variable at the given width.

Values for all Greeks and IV were multiplied by 100 ((i.e. Delta of 0.4 becomes 40$)$

Average partial effects of our logistic model are plotted in Figure 2. For robustness, we include in the appendix the marginal effects of the logistic model across a wide variety of quantiles, all of which yield similar results to our average partial effects estimates.

\section{FIGURE 2}

\section{LOGISTIC MODEL: SPECIFICATION 8}

\section{Logistic Model: Specification 8}

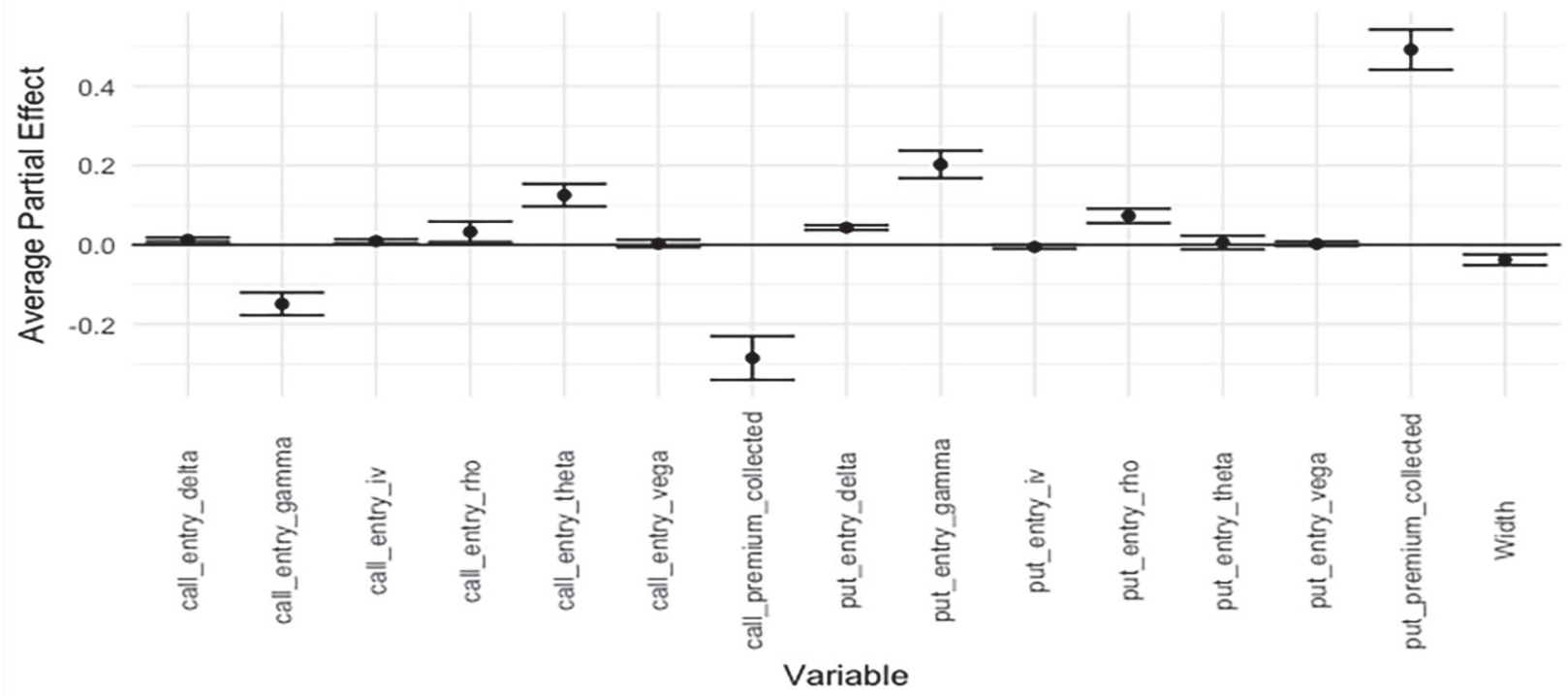

Figure 2: Average partial effects reported from our logistic model estimate in specification 8. Call and Put premiums collected have the largest measured effects on estimating the likelihood of profitability of strangles. The higher option premiums collected provide a cushion against potential losses. 


\section{Strategy Performance}

Profitability of the call and put options in the strangle positions are estimated by taking the difference between the bid price on entry and the ask price on the day of expiration. We calculate the annualized return on a given position by comparing the profit/loss on one contract to four times the initial margin required to take on the position. Across our out-of-sample test set of 7,548 positions, the average annualized return for all positions is $11.08 \%$ and an average annualized maximum drawdown of $-11.02 \%$. Using the BSM extension to only take on strangles that have an above average likelihood of profitability leads to a reduction in the average return to $9.17 \%$ but also reduces the average annualized maximum drawdown to $-9.31 \%$. Our baseline extension of the BSM framework correctly predicts profitability, across all strangle widths ${ }^{7}$, approximately $67 \%$ of the time. All of the estimated models are successful at having a lower average drawdown than the overall average drawdown across all positions within the test set.

The linear probability models are indeed successful at finding strangles that have a higher annualized return than the average within the test set, with specification 3-8 exceeding the overall test set average return. The logistic regression models are able to draw a marginally better decision boundary between profitable and losing strangles, causing the average maximum drawdown of the logistic model to beat the overall test set marginally at $-9.64 \%$. The logistic model is seemingly able to avoid many occurrences of small losses as indicated by the superior accuracy rate of predicting losing strangle positions, with the best of the logistic models in specification 8 , able to accurately predict $87 \%$ of losing strangles.

TABLE 9

MODEL PERFORMANCE 40-60 DTE

\begin{tabular}{|c|c|c|c|c|c|c|c|c|c|c|}
\hline Method & Model & 이 0 & 이 1 & 0 Accuracy & $1 \mid 0$ & $1 \mid 1$ & 1 Accuracy & Total Accuracy & Return & Max Drawdown \\
\hline Linear Probability Model & 1 & 740 & 410 & $64.35 \%$ & 1749 & 4649 & $72.66 \%$ & $71.40 \%$ & $8.97 \%$ & $-10.17 \%$ \\
\hline Linear Probability Model & 2 & 810 & 340 & $70.43 \%$ & 1893 & 4505 & $70.41 \%$ & $70.42 \%$ & $9.79 \%$ & $-10.13 \%$ \\
\hline Linear Probability Model & 3 & 874 & 276 & $76 \%$ & 2091 & 4307 & $67.32 \%$ & $68.64 \%$ & $11.25 \%$ & $-10.01 \%$ \\
\hline Linear Probability Model & 4 & 880 & 270 & $76.52 \%$ & 2117 & 4281 & $66.91 \%$ & $68.38 \%$ & $11.27 \%$ & $-9.90 \%$ \\
\hline Linear Probability Model & 5 & 879 & 271 & $76.43 \%$ & 2115 & 4283 & $66.94 \%$ & $68.39 \%$ & $12.22 \%$ & $-10.06 \%$ \\
\hline Linear Probability Model & 6 & 875 & 275 & $76.09 \%$ & 2080 & 4318 & $67.49 \%$ & $68.80 \%$ & $12.30 \%$ & $-10.03 \%$ \\
\hline Linear Probability Model & 7 & 882 & 268 & $76.70 \%$ & 2108 & 4290 & $67.05 \%$ & $68.52 \%$ & $12.23 \%$ & $-9.97 \%$ \\
\hline Linear Probability Model & 8 & 889 & 261 & $77.30 \%$ & 2156 & 4242 & $66.30 \%$ & $67.98 \%$ & $12.37 \%$ & $-9.95 \%$ \\
\hline Logistic Regression & 1 & 990 & 160 & $86.09 \%$ & 3422 & 2976 & $46.51 \%$ & $52.54 \%$ & $16.82 \%$ & $-10.00 \%$ \\
\hline Logistic Regression & 2 & 968 & 182 & $84.17 \%$ & 2783 & 3615 & $56.50 \%$ & $60.72 \%$ & $10.57 \%$ & $-9.45 \%$ \\
\hline Logistic Regression & 3 & 976 & 174 & $84.87 \%$ & 2815 & 3583 & $56 \%$ & $60.40 \%$ & $11.78 \%$ & $-9.55 \%$ \\
\hline Logistic Regression & 4 & 977 & 173 & $84.96 \%$ & 2835 & 3563 & $55.69 \%$ & $60.15 \%$ & $11.59 \%$ & $-9.34 \%$ \\
\hline Logistic Regression & 5 & 986 & 164 & $85.74 \%$ & 2830 & 3568 & $55.77 \%$ & $60.33 \%$ & $13.38 \%$ & $-9.65 \%$ \\
\hline Logistic Regression & 6 & 986 & 164 & $85.74 \%$ & 2785 & 3613 & $56.47 \%$ & $60.93 \%$ & $13.86 \%$ & $-9.76 \%$ \\
\hline Logistic Regression & 7 & 988 & 162 & $85.91 \%$ & 2837 & 3561 & $55.66 \%$ & $60.27 \%$ & $13.70 \%$ & $-9.76 \%$ \\
\hline Logistic Regression & 8 & 990 & 160 & $86.09 \%$ & 2873 & 3525 & $55.10 \%$ & $59.82 \%$ & $13.76 \%$ & $-9.64 \%$ \\
\hline BSM & & 888 & 262 & $77.22 \%$ & 2246 & 4152 & $64.90 \%$ & $66.77 \%$ & $9.17 \%$ & $-9.31 \%$ \\
\hline
\end{tabular}

Note: Out of sample results reported on $25 \%$ of data randomly selected prior to model fitment. Actual|Predicted. Test sample size: 7548; Test Total Return: 11.08\%; Test Max Drawdown: -11.02\%

Returns calculated by assuming four times initial margin is invested.

\section{CONCLUSION}

In our studies, we set out to empirically test the profitability of a non-directional option trading strategy by shorting SPY option strangles. Our results show that these strategies are generally profitable across all time frames and widths even during the critical crisis year of 2008. In fact, 2009 showed by far the largest average returns of $18.28 \%$ followed by $16.85 \%$ in 2011 . From a specific strategy perspective, the short strangle positions with a width of $10 \%$ had the largest average returns of over $23 \%$ in 2009 . This may imply that fear of a continuation of the crisis persisted far longer than actual price returns of the 
underlying market would suggest. In other words, option prices were inflated due to the heightened expectation of additional market crashes, driving up the prices of options. Therefore, option sellers were able to capitalize on the fear-induced long memory in option prices. Having said that, short selling strategies are invariably risky and our strategies are no exception. The trades post positive returns albeit with high variability and large average drawdowns of up to $-12.16 \%$.

In our fully specified linear probability model with an out-of-sample test of over 7,000 positions, we achieve an average annualized return of over $12 \%$ albeit with an average maximum drawdown of $-9.95 \%$ across positions. Our BSM extension that tests only strangles with an above average likelihood of profitability leads to a reduction in returns to about $9.2 \%$ but still generates a similar maximum drawdown. The BSM extension correctly predicts profitability about $67 \%$ of the time across all strangle widths ( $77 \%$ of losing strangles and $65 \%$ of winning strangles). Our fully specified logistic model yields the highest performance by correctly classifying $86 \%$ of losing strangles and $55 \%$ of winning strangles, corresponding to an average annualized return of nearly $14 \%$.

In closing, our studies show that shorting strangles can be profitable. However, given the high variability of returns and the potentially large drawdowns, these trades require a carefully crafted risk management strategy to avoid being "steam-rolled."

In future versions of this research we will re-examine the time frames to assess optimal days to expiration below and above our current target of 40-60 days. Furthermore, we may use more advanced statistical modeling techniques to predict the probability of in conjunction with the probability of profit.

\section{ENDNOTES}

1. We use $75 \%$ of our data (training sample) for model estimation and test our model on the remaining $25 \%$ of our data (test sample).

2. A substantial amount of time in our sample is plagued by artificially low interest rates as a result of the monetary stimulus following the financial crises in 2008 .

3. Generally, options that have strike prices beyond $+/-15 \%$ of the underlying price, that expire in $40-60$ days, have very little trading volume and prices near zero.

4. When a model is estimated on data with imbalanced classes the model becomes susceptible to always predicting the most frequently occurring class; classes in our test data are kept untouched to capture realworld frequencies. We address imbalanced classes by up-weighting losses which occur infrequently and by down-weighting profits which occur frequently.

5. Four times the initial margin is assumed to assure that each position taken on in the simulations has adequate capital to be funded.

6. In our data set, we express width as the percentage distance of a strike price from the underlying asset price at the time of entering the trade.

7. In our test set that was randomly selected from our full dataset we have $6041 \%, 5822 \%, 5793 \%, 5614 \%$, 552 5\%, 544 6\%, 475 7\%, 461 8\%, 419 9\%, 374 10\%, 350 11\%, 306 12\%, 267 13\%, 216 14\%, and 189 $15 \%$ strangles. 


\section{REFERENCES}

Black, F., \& Scholes, M. (1972). The Valuation of Option Contracts and a Test of Market Efficiency. The Journal of Finance, 27(2), 399. doi:10.2307/2978484.

Bollen, N. P. B., \& Whaley, R. E. (2004). Does Net Buying Pressure Affect the Shape of Implied Volatility Functions? The Journal of Finance, 59(2), 711-53. doi:10.1111/j.15406261.2004.00647.x.

Bondarenko, O. (2014). Why Are Put Options so Expensive? Quarterly Journal of Finance, 4(3), 1450015. doi:10.1142/S2010139214500153.

CBOE: Individual Index and Etf Volume Reports. (2017). Retrieved from

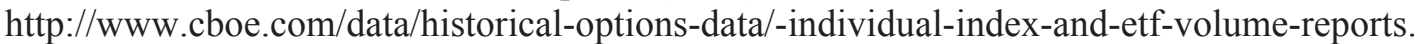

Chaput, J. S., \& Ederington, L. H. (2005). Volatility Trade Design. Journal of Futures Markets, 25(3), 243-79. doi:10.1002/fut.20142.

Coval, J. D., \& Shumway, T. (2001). Expected Option Returns. The Journal of Finance, 56(3), 983-1009. doi:10.1111/0022-1082.00352.

Dichtl, H., \& Drobetz, W. (2011). Portfolio Insurance and Prospect Theory Investors: Popularity and Optimal Design of Capital Protected Financial Products. Journal of Banking \& Finance, 35(7), 1683-97. doi:https://doi.org/10.1016/j.jbankfin.2010.11.012.

Ederington \& Guan. (2002). Why Are Those Options Smiling? The Journal of Derivatives, 10(2). Institutional Investor Journals Umbrella: 9-34. doi:10.3905/jod.2002.319193.

Kownatzki, C. (2016). How Good Is the VIX as a Predictor of Market Risk. Journal of Accounting and Finance, 16(6), 39-60.

Li, W-X., French, J. J., \& Chia-Sheng Chen, C. (2017, June). Informed Trading in S\&P Index Options? Evidence from the 2008 Financial Crisis. Journal of Empirical Finance, 42, 40-65. https://doi.org/10.1016/j.jempfin.2017.01.001.

Lowenstein, R. (2000). When Genius Failed: The Rise and Fall of Long-Term Capital Management. Paperback ed. New York, NY: Random House Trade Paperbacks.

Merton, R. C. (1973). Theory of Rational Option Pricing. The Bell Journal of Economics and Management Science, 4(1), 141. doi:10.2307/3003143.

Simon, D. P. (2007). An Examination of Short QQQ Option Trades. Journal of Futures Markets, 27(8), 739-70. doi:10.1002/fut.20265.

Tversky, A., \& Kahneman, D. (1992). Advances in Prospect Theory: Cumulative Representation of Uncertainty. Journal of Risk and Uncertainty, 5(4). Springer: 297-323. http://www.jstor.org/stable/41755005.

White, H. (1980). A Heteroskedasticity-Consistent Covariance Matrix Estimator and a Direct Test For Heteroskedasticity. Econometrica, 48(4), 817-38.

https://EconPapers.repec.org/RePEc:ecm:emetrp:v:48:y:1980:i:4:p:817-38. 


\section{APPENDIX}

\section{A Word on Commissions...}

We do not consider commissions in our studies for the following reasons: a) a number of researchers use a similar approach (Bakshi and Kapadia, 2003; Coval and Shumway, 2001; Bollen and Whaley, 2004); b) commissions costs today are about half of what they were 10 years ago with a trend towards next to zero commissions. Some brokerage firms today have started charging commissions only upon entry; c) professional option traders do not enter into positions with market orders but instead place a limit order typically at the average bid-ask price. Unlike other researchers who enter and exit positions at the midpoint of the closing bid-ask spread (Simon 2007), we enter our short strangle positions at the bid side and close the positions at the ask of the closing price. Therefore, we invariably incur a transaction cost that may be equivalent to typical commissions charged by brokerage firms.

\section{American Versus European Style Options}

European-style options can only be exercised at expiration whereas American-style options can be exercised on any business day up to and including on the expiration date. SPY options are Americanstyle. http://www.cboe.com/products/options-on-single-stocks-and-exchange-traded-products/options-onexchange-traded-products/options-on-etps-specs

The original BSM framework applies to European-style options only. Although numerous models have been developed to account for a slight premium in American-style options over European style options, we make a simplifying assumption in our theoretical treatment of probability of profit by relying on the BSM model. The rationale here is two-fold: a) We are not estimating option prices but rather evaluate the market-driven option prices that have the premium of the possibility of early exercise already built-in and b) our approach is conservative because it results in a slight underestimation of the probability of profit since the market prices of Calls $\left(P_{c}\right)$ and Puts $\left(P_{p}\right)$ in our probability model should theoretically be greater than European-style options and would therefore lead to an underestimation of the probability of profit.

\section{Margin Requirements}

Margin requirement for options are based on Chicago Board Options Exchange (CBOE) Margin Rules. The rules are set out in the CBOE Margin Manual dated April 2000. http://www.cboe.com/framed/ pdfframed?content=/learncenter/pdf/margin2-00.pdf\&section=SECT_MINI_SITE\&title=Cboe + Margin + Manual

For a Short Strangle position, the margin requirement is the greater of the requirement on the short put or call, plus the option proceeds on the other side. The initial margin for a short call (short put) is $100 \%$ of the short call (short put) proceeds plus $20 \%$ of the underlying asset value minus the out-of-the money amount if any. For a strangle, the proceeds from both option sales may be applied to the initial margin requirement. We use the $\mathrm{CBOE}$ definition of margins for all of our simulated returns. More precisely, we assume that an investor holds four times the initial margin amount for every position taken on to satisfy any capital constraints. 
TABLE 10

CALL DELTA ON ENTRY 40-60 DTE

\begin{tabular}{|c|c|c|c|c|c|c|}
\hline \hline Width & Min. & 1st Qu. & Median & Mean & 3rd Qu. & Max. \\
\hline $1 \%$ & 0.234 & 0.370 & 0.411 & 0.407 & 0.448 & 0.541 \\
\hline $2 \%$ & 0.102 & 0.274 & 0.326 & 0.325 & 0.382 & 0.526 \\
\hline $3 \%$ & 0.049 & 0.188 & 0.246 & 0.252 & 0.317 & 0.506 \\
\hline $4 \%$ & 0.028 & 0.118 & 0.174 & 0.191 & 0.261 & 0.485 \\
\hline $5 \%$ & 0.016 & 0.070 & 0.114 & 0.143 & 0.204 & 0.465 \\
\hline $6 \%$ & 0.011 & 0.045 & 0.082 & 0.113 & 0.163 & 0.443 \\
\hline $7 \%$ & 0.002 & 0.030 & 0.058 & 0.091 & 0.132 & 0.434 \\
\hline $8 \%$ & 0.002 & 0.021 & 0.041 & 0.073 & 0.103 & 0.423 \\
\hline $9 \%$ & 0.001 & 0.016 & 0.032 & 0.060 & 0.080 & 0.402 \\
\hline $10 \%$ & 0.000 & 0.013 & 0.027 & 0.050 & 0.066 & 0.377 \\
\hline $11 \%$ & 0.000 & 0.011 & 0.024 & 0.046 & 0.061 & 0.356 \\
\hline $12 \%$ & 0.000 & 0.010 & 0.025 & 0.044 & 0.058 & 0.362 \\
\hline $13 \%$ & 0.000 & 0.010 & 0.026 & 0.044 & 0.058 & 0.341 \\
\hline $14 \%$ & 0.000 & 0.010 & 0.027 & 0.043 & 0.057 & 0.313 \\
\hline $15 \%$ & 0.000 & 0.010 & 0.029 & 0.044 & 0.059 & 0.280 \\
\hline
\end{tabular}

TABLE 11

PUT DELTA ON ENTRY 40-60 DTE

\begin{tabular}{|c|c|c|c|c|c|c|}
\hline Width & Min. & 1st Qu. & Median & Mean & 3rd Qu. & Max. \\
\hline $1 \%$ & -0.518 & -0.436 & -0.412 & -0.410 & -0.385 & -0.287 \\
\hline $2 \%$ & -0.482 & -0.384 & -0.350 & -0.348 & -0.313 & -0.213 \\
\hline $3 \%$ & -0.447 & -0.337 & -0.296 & -0.297 & -0.255 & -0.151 \\
\hline $4 \%$ & -0.417 & -0.296 & -0.249 & -0.254 & -0.209 & -0.117 \\
\hline $5 \%$ & -0.412 & -0.261 & -0.208 & -0.217 & -0.168 & -0.093 \\
\hline $6 \%$ & -0.396 & -0.232 & -0.181 & -0.190 & -0.144 & -0.075 \\
\hline $7 \%$ & -0.376 & -0.207 & -0.159 & -0.170 & -0.125 & -0.061 \\
\hline $8 \%$ & -0.352 & -0.187 & -0.141 & -0.152 & -0.108 & -0.048 \\
\hline $9 \%$ & -0.338 & -0.167 & -0.123 & -0.134 & -0.091 & -0.041 \\
\hline $10 \%$ & -0.319 & -0.149 & -0.109 & -0.119 & -0.078 & -0.034 \\
\hline $11 \%$ & -0.306 & -0.135 & -0.096 & -0.108 & -0.068 & -0.030 \\
\hline $12 \%$ & -0.302 & -0.123 & -0.088 & -0.099 & -0.061 & -0.025 \\
\hline $13 \%$ & -0.285 & -0.114 & -0.081 & -0.093 & -0.056 & -0.023 \\
\hline $14 \%$ & -0.269 & -0.109 & -0.079 & -0.089 & -0.055 & -0.017 \\
\hline $15 \%$ & -0.256 & -0.109 & -0.078 & -0.086 & -0.053 & -0.019 \\
\hline
\end{tabular}



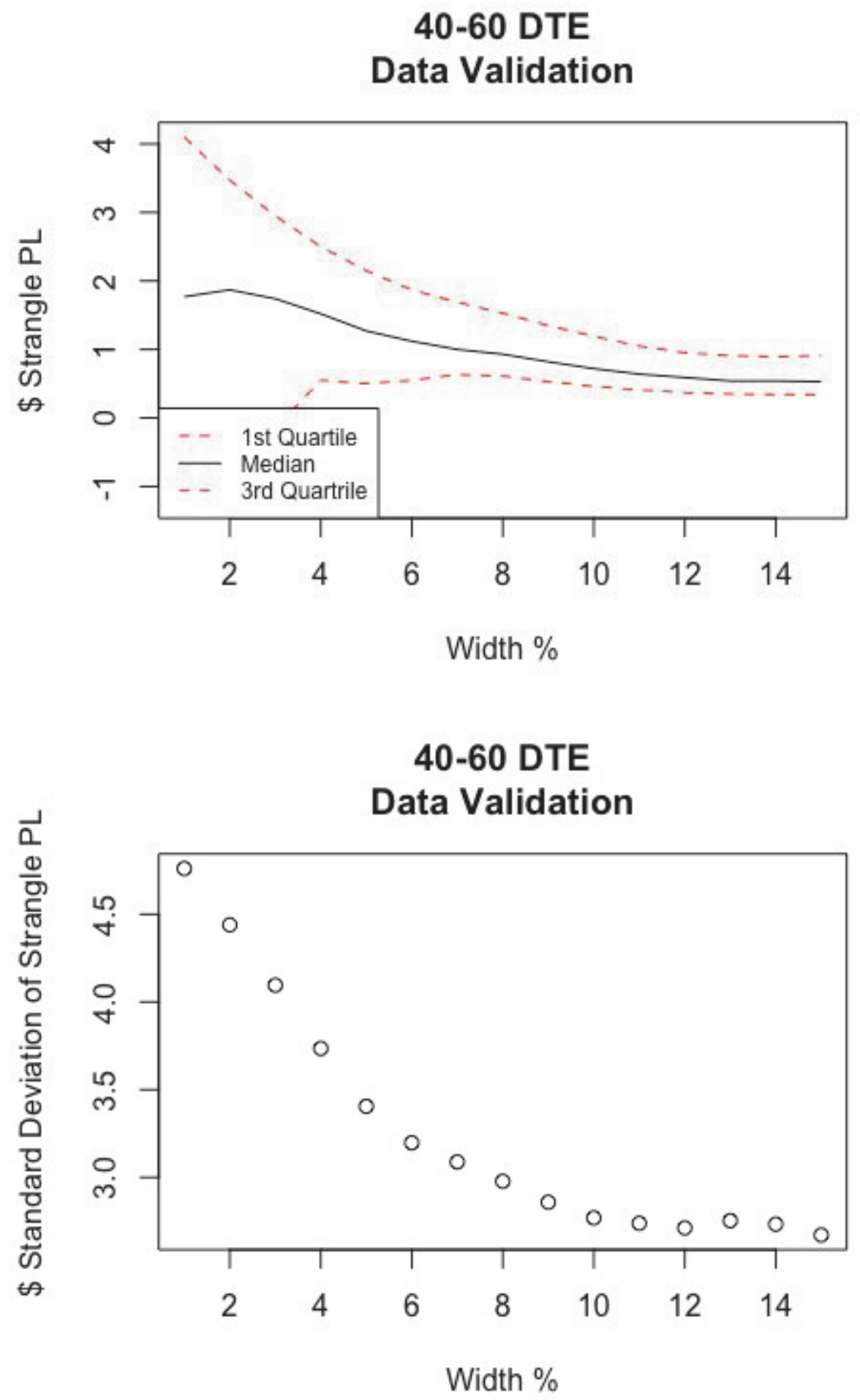


\section{Logistic Model: Specification 8}

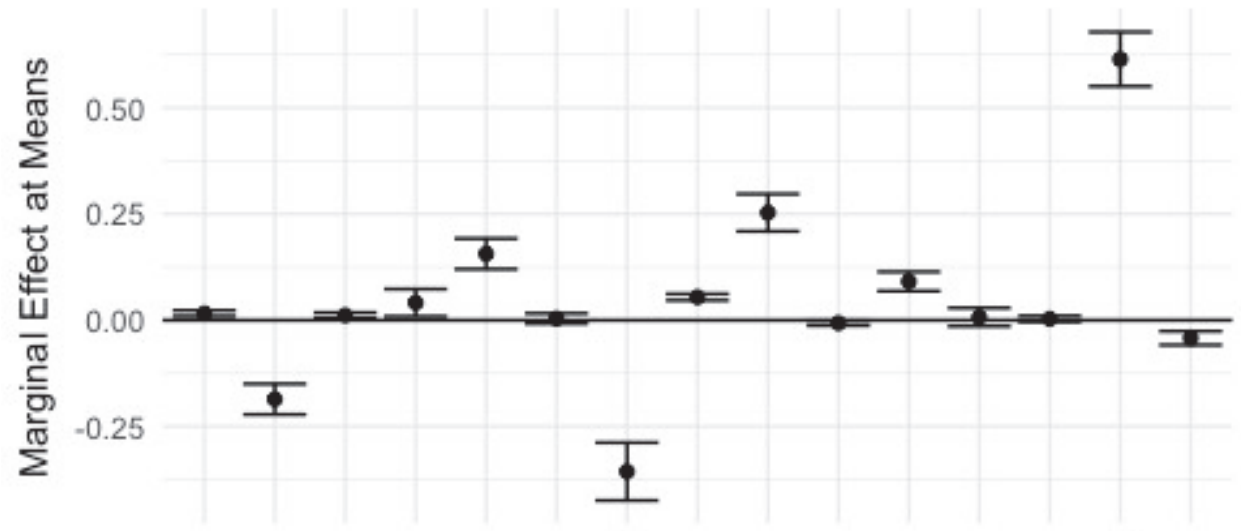

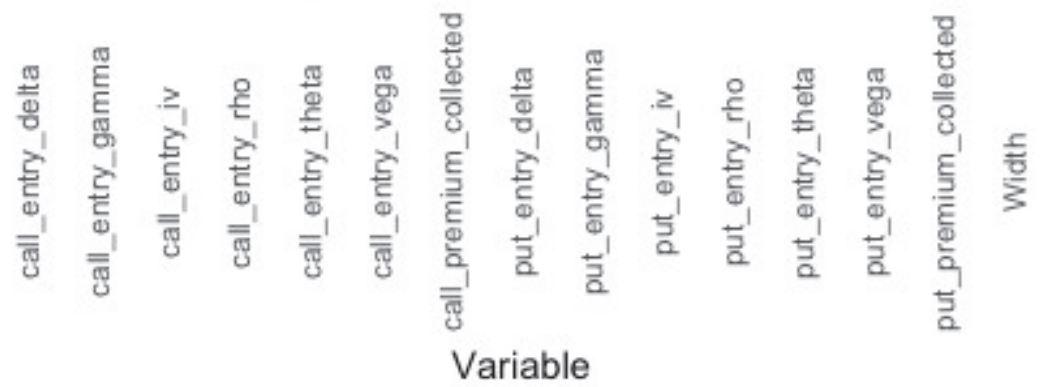

\section{Logistic Model: Specification 8}

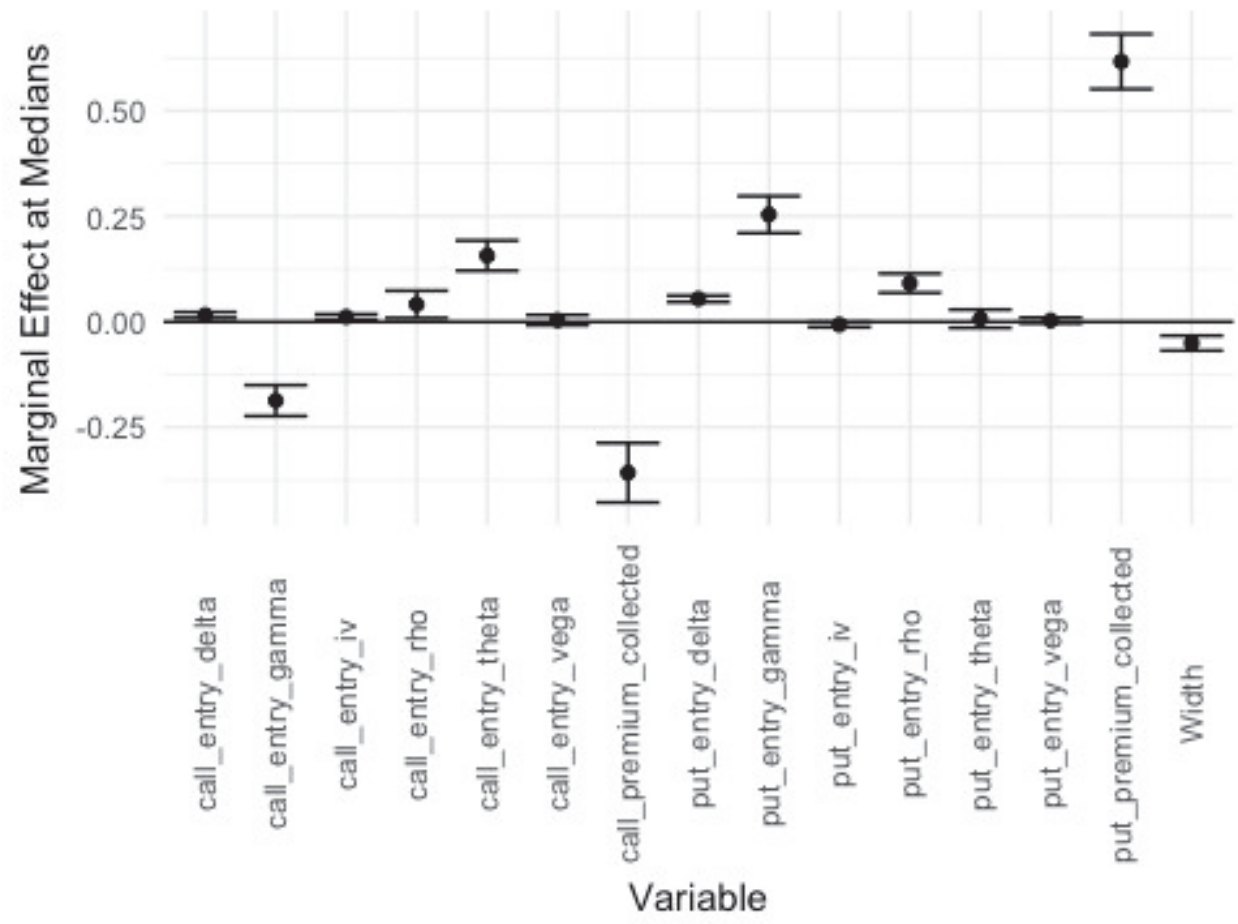



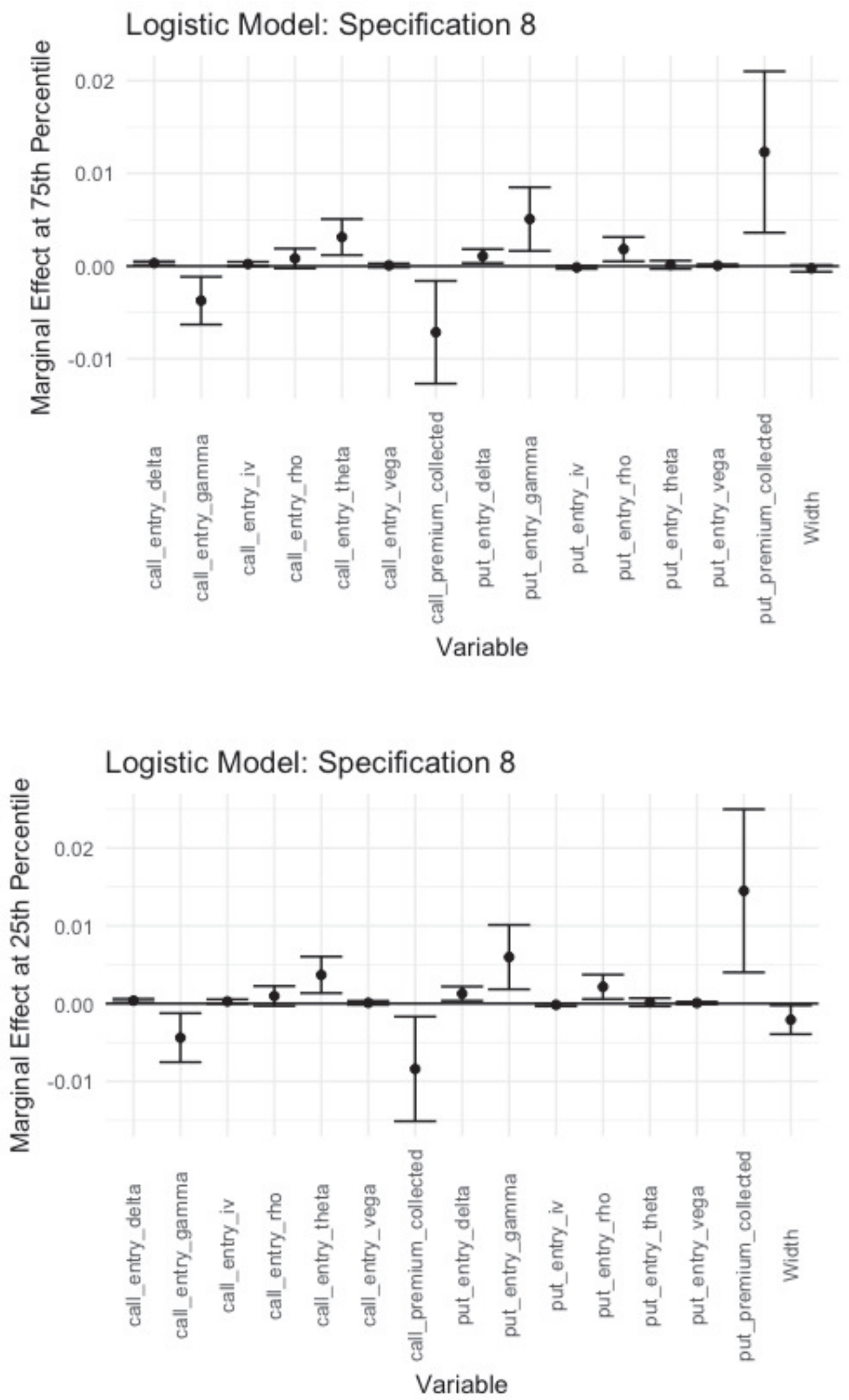